\title{
Numerical Simulation of Neutral Buoyancy Experiment for Spacecraft
}

\author{
Liu Shuhan ${ }^{1,2, a)}$, Zhu Zhanxia ${ }^{3,4, b)}$ \\ ${ }^{1}$ College of Aeronautics, Northwestern Polytechnical University, Xi'an 710072 China. \\ ${ }^{2}$ National key laboratory of aerospace flight dynamics, Xi'an 710072, China. \\ ${ }^{3}$ College of Aeronautics, Northwestern Polytechnical University , Xi'an 710072 China. \\ ${ }^{4}$ National key laboratory of aerospace flight dynamics, Xi'an 710072, China.

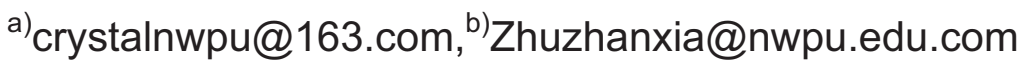

\begin{abstract}
Keywords: Spacecraft; Neutral buoyancy; Computational Fluid Dynamics; Hydrodynamic coefficients
\end{abstract}

\begin{abstract}
With the success of Shenzhou spacecraft rendezvous and docking with Tiangong-1 and Tiangong-2, the future tasks of the space station such as space garbage clean-up requires the control of spacecraft attitude to be more and more accurate. Because of the high cost of the attitude control and regulation test in space, the experiments are usually carried out on the ground. In order to simulate the microgravity environment of space, the gravity of the aircraft model is needed to be overcame in the ground test. Presently, there are two ways that not only overcome the gravity of the aircraft model but also make the aircraft to freedom of movement: one is to use non-contact force to overcome the gravity, such as electromagnetic force, but this approach requires the control of non-contact force to be very accurate, which needs difficult technology and faces the high cost; another way is to use liquid buoyancy to overcome gravity, which only needs to ensure that the average density of the aircraft model is equal to the density of the liquid, and the aircraft model can be suspended at any position in the liquid, using the thrust produced by the propeller to carry out the free movement. This technique is not difficult and low cost, but in the control of aircraft model movement needs to overcome the force and torque generated by the liquid. Therefore, the buoyancy of water is used to overcome the gravity of the vehicle model, and a neutral buoyancy force is obtained. Then the numerical analysis technique is used to calculate the hydrodynamic coefficient of the model. At the same time, by changing the propeller thrust, the response curves of aircraft moving in vertical and horizontal directions are simulated and verified. The results show that the model can reach a new equilibrium state in $0.8 \sim 1$ seconds. This paper provides a theoretical basis for guiding the tunnel test of aircraft model, which is one of the important links in the iterative design of space vehicles.
\end{abstract}

\section{Introduction}

With the implementation of the space station plan and the space robot plan for cleaning up space debris, the requirements for spacecraft attitude control will become more accurate [1-4]. Therefore, it is necessary to obtain the relevant control parameters through the neutral buoyancy simulation experiment [6-8].

In the ground experiment, the neutral buoyancy experiment system with the same specific gravity density with water plays a very important role. The neutral buoyancy tank can be very large [9-11], which can be used for long-time and unlimited six-DOF continuous motion test of large-scale experiment body. It is an ideal space activity and operation demonstration simulation method. But first of all, we need to get the hydrodynamic coefficient of the experimental body to design the control law to control the experimental body. Therefore, it is necessary to carry out water tunnel experiment on the experimental body, which is not only costly but also laborious in the whole experiment.

With the development of computer technology, it is possible to analyze and calculate the hydrodynamics coefficient of the experiment body with numerical simulation technique [12-13]. 
Therefore, this paper uses the mature CFD technique to calculate the hydrodynamic coefficient of the experiment body, and then corrects the hydrodynamic coefficients to obtain the final hydrodynamic coefficients. This method reduces the number of experiments, which saves the cost and time while guaranteeing the accuracy of the hydrodynamic coefficient of the experimental body as well as improving the efficiency to obtain the hydrodynamic coefficient of the experimental body quickly.

Jiang et al. [14] used CFD to calculate the drag coefficients and pitch moment coefficients of cylinder, sphere and cube at different water velocities and angles of attack. However, the experimental model used was too simple to consider the experimental zone sideslip angle and shape change and so on.

Yang et al. [15] calculated the hydrodynamic derivative of a ship. However, the use of the ship model is very simple, thus the accuracy of the results is inadequate.

Zhang et al. [16] took a long-range submersible as a research object, using approximation estimation, potential flow theory based on the panel method, and FLUENT simulation of circulating water tank test respectively, to solve its hydrodynamic coefficient. The calculation results are compared with the experimental results of model circulating tank operation performance, and then the applicable range of the three calculation methods is summarized and evaluated. However, there is also the problem of over-simplification of the model.

In this paper, a spacecraft experiment with a mechanical manipulator is analyzed and calculated by numerical simulation. The hydrodynamic coefficients of the experiment body under different working conditions are calculated respectively when the manipulator is linear and L shape. The results are verified by simulation, which can be used to guide the experimental body to conduct water tunnel test and design the control system of spacecraft experiment body.

\section{Hydrodynamic coefficients calculation}

\subsection{Conditions and solving settings}

1) Geometric shape

Considering that the space mission of spacecraft is to clean up the space garbage, the manipulator makes various actions to grab the space garbage, therefore two typical motion states of the spacecraft robot arm: linear and L-shaped, are selected. For the CFD calculation, the engine is closed, and a number of small steps for smoothing, processing results shown in Fig. 1 and Fig. 2.

In order to describe the directions of the three force and torque coefficient, the following coordinate system are defined: $\mathrm{X}$ axis in the experimental body symmetry plane parallel to the robot arm pointing forward, $\mathrm{Y}$ axis perpendicular to the experimental body symmetry to the right, $\mathrm{Z}$ axis in the experimental body symmetry plane perpendicular to the XY plane pointing down, and the origin $\mathrm{O}$ is located in the experimental body of the geometric center, as shown in Fig. 1 and Fig. 2.

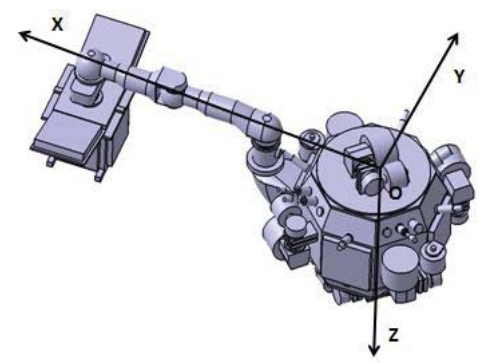

FIGURE 1. the mechanical arm is in a straight line 


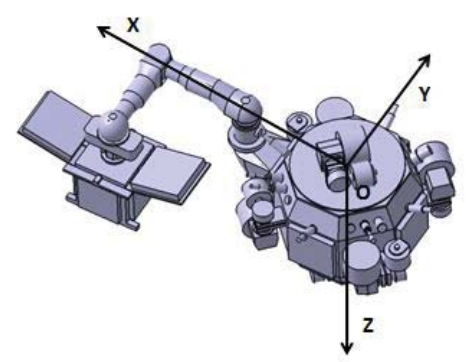

FIGURE 2.the mechanical arm is in a state of $\mathrm{L}$

2) Mechanical motion condition

The hydrodynamic coefficients of each state were calculated at $100(5 \times 5 \times 4)$ operating points as shown in Table 1, respectively.

TABLE 1. condition of the experimental body

\begin{tabular}{cccc}
\hline Numble & Fluid velocity $(\mathrm{m} / \mathrm{s})$ & Angle of attack $\left(^{\circ}\right)$ & Sideslip angle $\left(^{\circ}\right)$ \\
\hline 1 & 0.1 & 0 & 0 \\
2 & 0.2 & 5 & 5 \\
3 & 0.3 & 10 & 10 \\
4 & 0.4 & 20 & 20 \\
5 & 0.5 & 30 & - \\
\hline
\end{tabular}

3) Solving settings

Fluent software was used to calculate the hydrodynamic coefficients, with implicit 2D single precision pressure-based solver, $\mathrm{k}-\varepsilon$ turbulence model, and Green-Gauss Node Based for gradient, where the water density is $\rho=998.2 \mathrm{~kg} / \mathrm{m}^{3}$, the viscosity coefficient $\mu=0.001003 \mathrm{~kg} /(\mathrm{m} \cdot \mathrm{s})$, and the operating ambient pressure is $1 \mathrm{~atm}$.

2.2 Hydrodynamic coefficient

When the experimental device is moving in water, the drag coefficient and the pitch moment coefficient of the experimental body are very important to the movement and longitudinal attitude of the experimental body. Therefore, this paper analyzes the factors that influence the drag coefficient and the pitch moment coefficient.

It is found that the drag coefficient and the pitch moment coefficient do not change with the velocity of the water when the water velocity changes in a small range. The typical values are shown in Fig. 3 to Fig. 6.

The drag coefficient curve and the pitch moment coefficient curve of the manipulator in the linear and L state are corresponding to each other, which means that when the sideslip angle and the angle of attack are within a certain range, the state of the arm does not change the development of the drag coefficient and the pitch moment coefficient. But the state of the manipulator will affect the values of the drag coefficient and the pitch moment coefficient, as shown in Fig. 3 to Fig. 6. Whether the state of the manipulator will influence the development of the drag coefficient and the pitch moment coefficient at a larger angle of attack and a larger angle of attack will require further study.

As the angle of attack increases, the drag coefficient gradually decreases and then increases as shown in Fig. 3, 5, 7 and 9, while the pitch moment coefficient increases as the angle of attack increases as shown in Fig. 6, 8, and 10. As the angle of attack increases, the normal force acting in the Z-axis of the body coordinate system gradually increases, and the axial force acting on the $\mathrm{X}$-axis direction of the body coordinate system gradually decreases, but the normal force and axial force are at the $\mathrm{Z}$ direction, and the pressing force is in front of the reference point $\mathrm{O}$. Thereby it generates a rise torque, and the rise-up torque is gradually increased, that is, the pitch connection coefficient is gradually increased. At the same time, as the angle of attack increases, the viscous frictional resistance and differential pressure resistance of the experimental body decrease, so the drag coefficient decreases first, and then with the further increase of the angle of attack, the fluid begins to separate and rapidly increase. Thus the drag coefficient gradually increased, as shown in 
Fig. 3, 5, 7 and 9 .

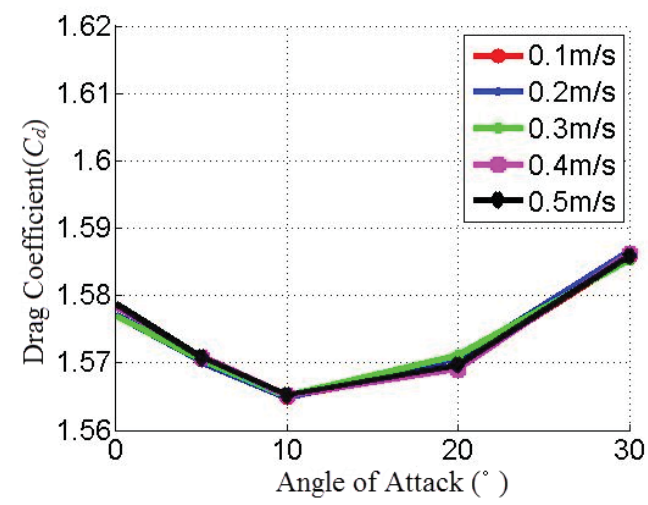

FIGURE 3. The relationship between the drag coefficient and the angle of attack (mechanical arm: linear, the sideslip angle: 0 degrees)

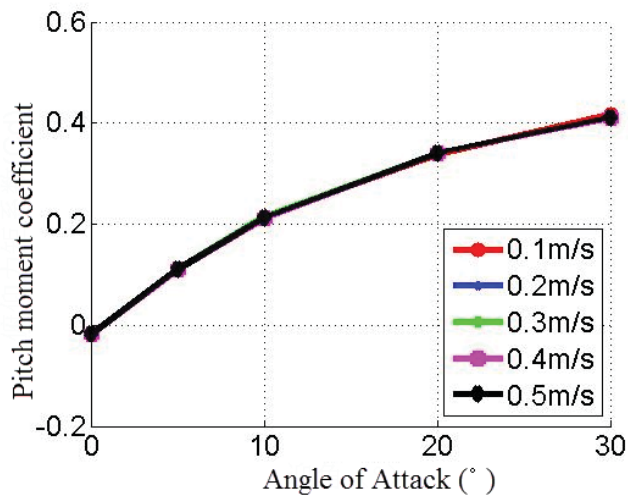

FIGURE 4. The relationship between the pitching moment coefficient and the angle of attack (mechanical arm: linear, the sideslip angle: 0 degrees)

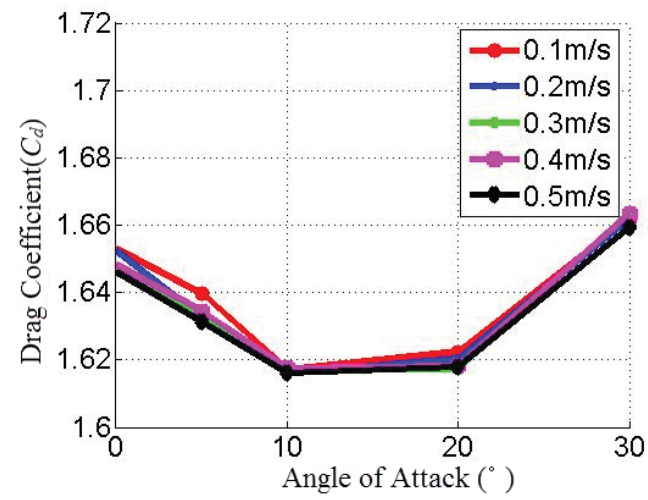

FIGURE 5. The relationship between the drag coefficient and the angle of attack (mechanical arm: L, the sideslip angle: 0 degrees)

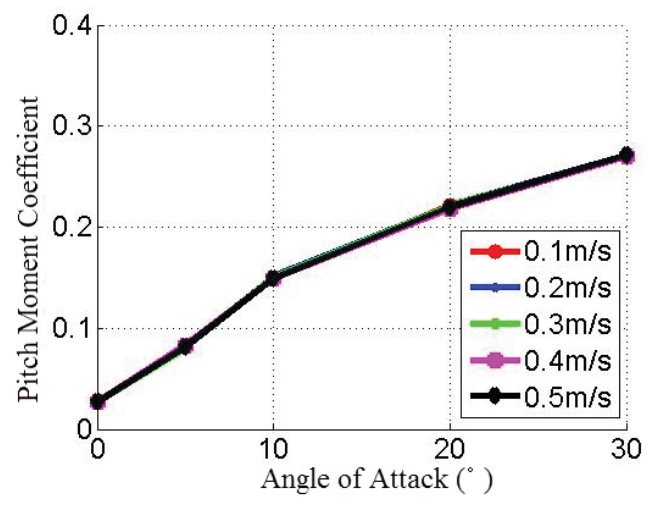

FIGURE 6. The relationship between the pitching moment coefficient and the angle of attack (mechanical arm: L, the sideslip angle: 0 degrees) 
When there is a small side slip angle, both sides of the robot will affect the drag coefficient and the pitch moment coefficient for both the straight line and the L-shape of the manipulator. However, the drag coefficient and the pitch moment coefficient vary with the angle of attack curve shape has little effect, as shown in Fig. 7 to Fig. 10.

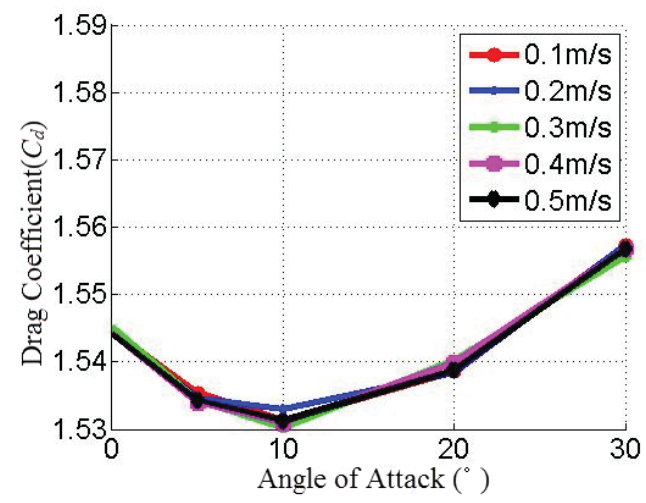

FIGURE 7. The relationship between the drag coefficient and the angle of attack (mechanical arm: linear,, the sideslip angle: 10 degrees)

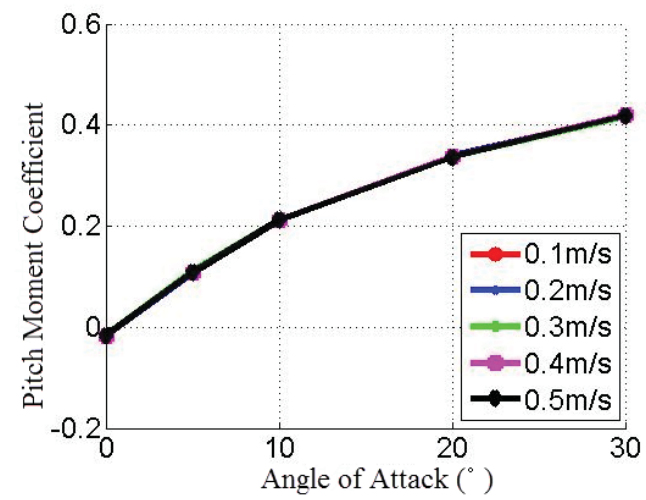

FIGURE 8. The relationship between the pitching moment coefficient and the angle of attack (mechanical arm: linear, the sideslip angle: 10 degrees)

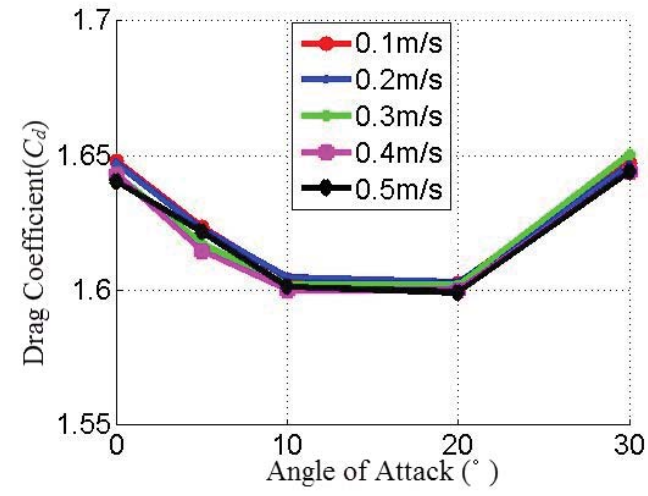

FIGURE9. The relationship between the drag coefficient and the angle of attack (mechanical arm: L, the sideslip angle: 10 degrees) 


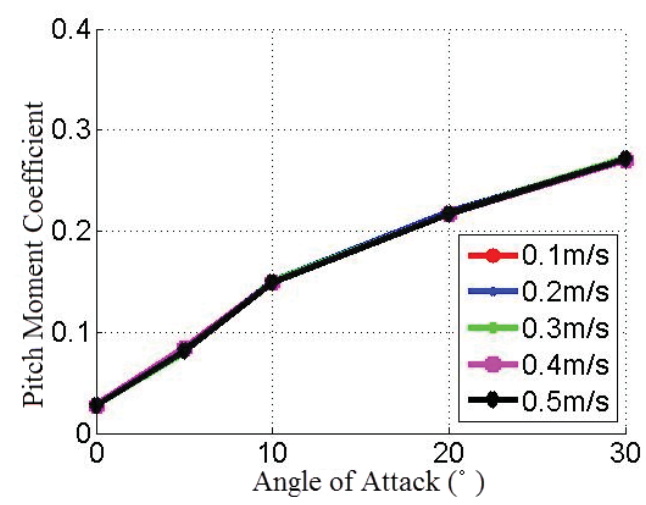

FIGURE 10.The relationship between the pitching moment coefficient and the angle of attack

(mechanical arm: L, the sideslip angle: 10 degrees)

\section{Dynamic simulation analysis}

The hydrodynamic coefficients calcula- ted by CFD can be used to simulate the dynamic model and simulate the motion of the experiment body. The response curve of the experiment body underwater under the given speed is verified, and the response time of the experiment body is obtained.

3.1 Dynamic modeling of experimental body

1) Modeling the in the vertical direction

Assuming that the body moves in the vertical direction and does not consider rotation, the equation is given by

Where $T_{z}$ is engine thrust, $\mathrm{F}$ is buoyancy, $\mathrm{G}$ is gravity, $\mathrm{Q}$ is dynamic pressure of water, $\mathrm{S}$ is reference area, $\mathrm{C}$ is drag coefficient in $\mathrm{Z}$ direction, $\mathrm{m}$ is experimental mass, $\mathrm{c}$ is damping coefficient, $\mathrm{k}$ is elastic coefficient, and $\mathrm{S}$ is the experimental body displacement. When moving slowly in water, $\mathrm{c}$ and $\mathrm{k}$ can be ignored.

2) Modeling the in the horizontal direction

Assuming that the experimental body moves only in the vertical direction and does not account for rotation, the equation is given by

$$
m s^{\prime \prime}+c s^{\prime}+k s=T_{x}+Q S C_{d x}
$$

Where, $T_{x}$ is the engine thrust, $\mathrm{Q}$ is the hydrodynamic pressure, $\mathrm{S}$ is the reference area, and $C_{d x}$ is the coefficient in the $\mathrm{X}$ direction drag.

3.2 Simulation of the experimental body

1) Simulation conditions

The density of water is $\rho=1000 \mathrm{~kg} / \mathrm{m}^{3}$, the mass of the experiment body is $m=30 \mathrm{~kg}$, the volume of the experimental body is $V=0.03 \mathrm{~m}^{3}$, and the reference area is $S=0.1 \mathrm{~m}^{2}$.

2) Vertical dynamic simulation

Take the thrust is $T_{z}=50 \mathrm{~N}, C_{d z}=1.6$ and the simulation results are shown in Fig. 11 and Fig. 12. 


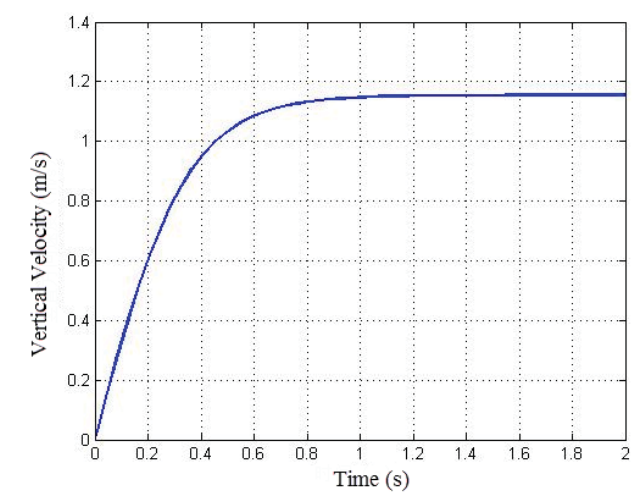

FIGURE1 1. The relationship between the vertical velocity and time

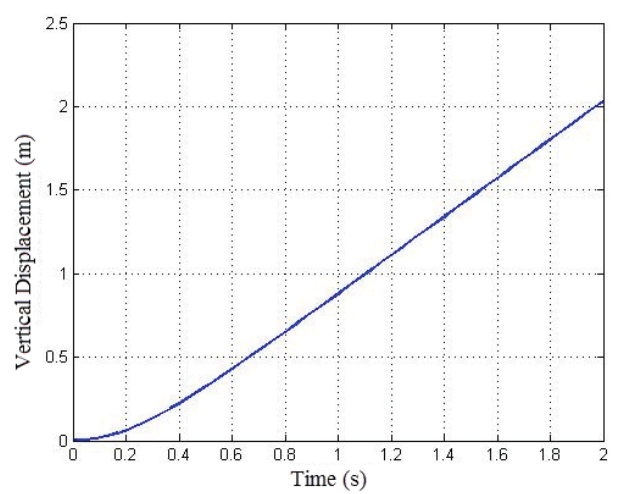

FIGURE 12. The relationship between the vertical displacement and time 3) Horizontal dynamic simulation

Take the thrust $T_{x}=100 N, C_{d x}=1.5$, and the simulation results are shown in Fig.13 and Fig. 14 .

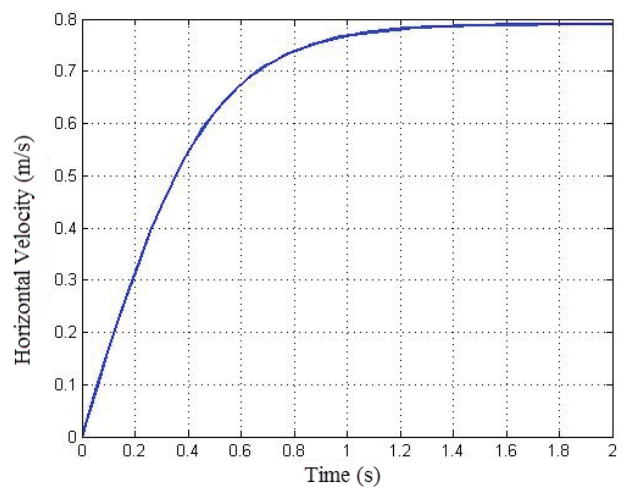

FIGURE 13. The relationship between the horizontal velocity and time

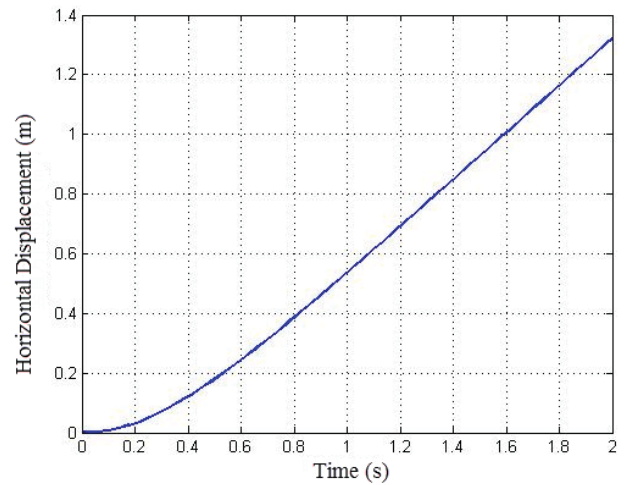

FIGURE 14. The relationship between the horizontal displacement and time

From the simulation curves of horizontal and vertical motion, we can get that, when the thrust force is given, the experiment body will do the acceleration and deceleration gradually. After a 
certain period of time, the resistance and thrust will be balanced. From the figures, it can be found that the velocity reaches $95 \%$ of the steady-state value for the first time in about 0.8 seconds, which means that the experimental body can quickly overcome the water resistance and achieve the desired steady-state value.

\section{Conclusion}

In this paper, the scaled experimental model of the spacecraft is established, and the micro step as well as the thruster is processed accordingly, which is made for the CFD simulation. The experimental results show that the hydrodynamic coefficients ( 3 force coefficients and 3 moment coefficients) of each state can be obtained by the numerical analysis of more than 100 states of the manipulator with linear and L shape. The influence of the velocity, angle of attack and sideslip angle on the drag coefficient and the pitching moment coefficient are compared and analyzed.

The experimental results show that the experimental model of the spacecraft can respond quickly and reach a new equilibrium state when the manipulator is linear or L-shaped. The hydrodynamic coefficients calculated by CFD can be simulated by establishing the dynamic model, which can guide the experiments to conduct water tunnel experiments. At the same time, the hydrodynamic coefficient calculated by CFD is used to design the control law, which lays a solid foundation for the design of autopilot.

After obtaining the experimental data of experimental cavity tunnel experiment, the theoretical data calculated by CFD should be compared with each other and modified with each other to accumulate experience for better CFD calculations and experiments.

\section{References}

[1] Guowei Xie.Study on the Space Robot Motion Control [D],Harbin,Harbin Institute of Technology,2006.(in Chinese)

[2] Di Zhang.Dynamics Parameter Identification and Control of Space Robot[D], Harbin,Harbin Institute of Technology,2013.(in Chinese)

[3] R Lampariello, G Hirzinger.Modeling and Experimental Design for the On-Orbit Inertial Parameter Identification of Free-Flying Space Robots.International Design Engineering Technical Conference \& Computer Information in Engineering Conference, 2005, 8(11):881-890.

[4] Lei Jin,Xu Shijie.Inertial Parameter Identification of Unknown Object Captured by a Space Robot[J].Journal of Astronautics, 2012, 33(11):1570-1576.(in Chinese)

[5] Zhixiang Chen. Weightless environment simulation techniques of neutral buoyancy [J]. Spacecraft Environment Engineering, 2000(1): 1-6.(in Chinese)

[6] Zhanxia Zhu. Construction of Microgravity Environment for Spacecraft Operation [M], China Astronautic Publishing House, 2013. (in Chinese)

[7] Cuiping Yue. Research of the High Precision Control System for Semi-Active Microgravity Ground Simulation Platform [D]. Hefei, University of Science and Technology of China, 2010. (in Chinese)

[8] Feng Hao.Research on Rotary-arm Micro-gravity simulation Device of Space Manipulator[D]. Harbin,Harbin Institute of Technology,2010. (in Chinese)

[9] Aijun Mai,Laijie Lu,Lei Zhang et al.American Neutral Buoyangcy Facility and Its Applications[J],Manned Spaceflight, 2012,18(4):19-25.(in Chinese)

[10] SJ Hoffman.Advanced EVA Capabilities: A Study for NASA's Revolutionary Aerospace Systems Concept Program[J].Science Applications International Corporation , NASA/TP-2004-212068.

[11] DL Akin, ML Bowden.EVA, robotic, and cooperative assembly of large space structures[J].Aerospace Conference, 2002, 7(7):7-3599-7-3610 .

[12] Wenhua Wang,Yanying Wang.Dynamic analysis for ship launching based on CFD 
method[J],Journal of Dalian Maritime University, 2009, 35(3):1-4. (in Chinese)

[13] Chunlei Yang,Renchuan Zhu,Guoping Miu.CFD-based numerical simulation of hull/propeller/rudder interaction[J],Chinese Journal of Hydrodynamics, 2011, 26(6):667-673. (in Chinese)

[14] Qiying Jiang,Qun Fang.Exploring Preliminarily Design and Simulation of the Shape for Experimental Object of Spacecraft Experiment of Neutral Buoyancy[J],Journal of Northwestern Polytechnical University, 2011, 29(5):751-756. (in Chinese)

[15] Bo Yang, Lin Wan, Xiao Wang et al.Calculation of ship hydrodynamic derivatives by CFD method [C]. Excellent Symposium of 2007 Annual Conference of China Maritime Society, 2007, 30-33. (in Chinese)

[16] He Zhang, Yongjie Pang, Yie Li.Study of AUV's Hydrodynamic Coefficients Calculation Methods[J],Journal of Wuhan University of Technology(Transportation Science \& Engineering), 2011, 35(1):16-18. (in Chinese) 